\title{
La Investigación Activista Feminista. Un diálogo metodológico con los movimientos sociales
}

\author{
Feminist activist research. A methodological dialogue with \\ social movements
}

\author{
Alejandra Araiza Díaz \\ Universidad Autónoma del Estado de Hidalgo, México \\ Universitat Rovira i Virgili de Tarragona \\ Robert GonzÁlez García \\ Universidad Autónoma del Estado de Hidalgo, México \\ rgonza67@gmail.com (MÉXICO)
}

Recibido: 23.012017

Aceptado: 18.08 .2017

\section{RESUMEN}

Este artículo revisa la estrategia de investigación activista en movimientos sociales, pensada desde la perspectiva de la epistemología feminista. Presenta análisis retrospectivos de las propias experiencias de Investigación Activista de los autores y las pone a la luz de un nuevo marco metodológico: la Investigación Activista Feminista (IAF). El texto parte de la propuesta de los "conocimientos situados" y sitúa la emergencia de la IAF en la confluencia de los nuevos movimientos globales y la tercera ola del feminismo. Concluimos que las experiencias de investigación activista en las que hemos participado se pueden incluir en la IAF y suponen la aproximación más adecuada desde las ciencias sociales al sujeto movimiento social tanto epistemológica como metodológicamente, ya que permiten recabar datos y análisis que devienen en conocimientos situados, académicamente relevantes y socialmente útiles.

\section{PALABRAS CLAVE}

Investigación activista, epistemología feminista, movimientos sociales, conocimientos situados, metodología cualitativa 


\begin{abstract}
This article reviews the activist research strategy in social movements, thought from the perspective of feminist epistemology. It presents retrospective analyzes of the authors' own Activist Research experiences and puts them in the light of a new methodological framework: Feminist Activist Research (FAR). The paper starts from the proposal of the «situated knowledge» and places the emergence of the FAR at the confluence of the new global movements and the third wave of feminism. We conclude that the activist research experiences in which we have participated can be included in the FAR and suppose the most adequate approach from the social sciences to the subject social movement both epistemologically and methodologically, since they allow us to collect data and analysis that become as situated knowledge, academically relevant and socially useful.
\end{abstract}

\title{
KEY WORDS
}

Activist research, feminist epistemology, social movements, situated knowledge, qualitative methodology

\section{INTRODUCCIÓN}

En las últimas décadas, han ido proliferando las críticas a la ciencia positivista, esa que promueve la distancia y la desafección respecto a los objetos de estudio. Una de estas vertientes críticas -que nos ha ido interpelando a lo largo del tiempo- proviene de las epistemologías feministas, no sólo en el sentido de cuestionar el sesgo de género en la ciencia (empirismo feminista) o de promover la mirada desde los márgenes (punto de vista feminista), sino de apostar -con Haraway (1991)- por el ejercicio del posicionamiento, por hacer visible el lugar desde el cual miramos.

El problema -según afirman Martínez, Biglia, Luxán, Fernández, Azpiazu y Bonet (2014)- es que se mantiene la idea de que las propuestas de las epistemologías feministas son más fácilmente abarcables en el plano teórico que en el plano empírico. Disponemos, hoy en día, de más reflexiones epistemológicas, pero todavía no hay un consenso sobre los métodos propiamente feministas.

La ciencia, como empresa de la modernidad, tuvo desde sus inicios ciertas formas de hacer que continúan siendo practicadas en la actualidad. Algunas de estas formas son y han sido cuestionadas por las feministas, pues se enmarcan dentro del androcentrismo. Otras aparecen frente a estas teóricas como irrenunciables. Así, pareciera que el quehacer científico requiere, por un lado, de la objetividad; $y$, por otro, de una comunidad de pares para validar nuestros conocimientos en común. Estas líneas están envueltas en esa idea de comunidad científica. No se trata tanto de exponer los datos provenientes de diferentes 
investigaciones, sino de construir un diálogo -a partir de ellas- sobre los métodos que utilizamos y las formas en que podemos reinventarlos.

El artículo parte de las reflexiones conjuntas acerca de la investigación activista en movimientos sociales, pensadas desde la perspectiva de la epistemología feminista. Presenta el ejercicio que realizamos el autor y la autora sobre nuestras respectivas experiencias de Investigación Activista en Barcelona, a partir de las cuales nos parece más adecuado seguir las directrices de la Investigación Activista Feminista (IAF). El texto busca explicar el recorrido de estas reflexiones.

Así, el primer apartado aborda la propuesta epistemológica de Donna Haraway (1991) conocida como "conocimientos situados", pues es la perspectiva epistemológica que creemos más adecuada para el uso de las metodologías que exponemos. El segundo apartado se centra en las nuevas realidades y subjetividades que emergen a la par del movimiento altermundista y de la tercera ola del feminismo, las cuales son afines tanto para los sujetos de conocimiento como para los sujetos cognoscentes. El tercer apartado plantea un vínculo entre la epistemología feminista, en concreto la idea de conocimientos situados, y la IAF. Creemos que este tipo de investigación feminista ofrece posibilidades más cuidadas y situadas epistemológicamente para realizar Investigación Activista en general. El cuarto apartado presenta los casos en los que las investigadoras hemos participado, así como las reflexiones que hemos elaborado a partir de ellas. Por último, cerramos con unas conclusiones que insisten en la importancia de seguir reflexionando sobre estos temas desde la academia.

\section{CONOCIMIENTOS SITUADOS}

Donna Haraway, considerada una de las precursoras de la epistemología feminista posmoderna, lanzó una propuesta denominada "conocimientos situados". A diferencia del punto de vista feminista, que se centraba en la experiencia de las mujeres y el privilegio de mirar desde los márgenes, Haraway (1991) insistía en que el mero ejercicio de posicionamiento es el que proporcionaría la objetividad.

En efecto, Haraway -al igual que otras teóricas feministas- no renuncia a la idea de objetividad. Al contrario, como alternativa a la ciencia androcéntrica o a la salida relativista cínica, propone: "los conocimientos parciales, localizables y críticos, que admiten la posibilidad de conexiones llamadas solidaridad en lo político y conversaciones compartidas en la epistemología" (Haraway, 1991: 329). Ella considera que es a partir de la parcialidad, y no de la universalidad, que se logra el conocimiento racional. Cuando Haraway habla de conocimientos situados, debería añadirse el apellido "conectados parcialmente", pues no basta con localizar el conocimiento o con ser crítico; necesitamos un circuito universal de conexiones, basado en la habilidad parcial de traducir (Araiza, 2013). Ella intenta alejarse de la idea trascendental del yo-investigador y busca en la parcialidad una objetividad. Entiende que es necesario conectar con otras en ese ejercicio. Se trata -dice- de unirse al otro, de ver junto al otro, sin pretender ser el 
otro. Asimismo, Haraway -como se puede ver en trabajos posteriores- acentúa la potencialidad de las comunidades científicas. Es por ello que habla de un circuito universal de conexiones. Usa la metáfora (Haraway, 1997) del "testigo modesto" para describir una práctica instaurada por la Royal Society of London, la cual describe al científico mostrando sus hallazgos frente a una comunidad de pares. Ella critica las formas de hacer de esta comunidad tradicional, pero nos invita a no renunciar a la potencia de validar nuestros conocimientos colectivamente. En ese texto termina abogando por un "testigo modesto mutado". Esta mutación ya se advertía aquí con la idea de conexiones parciales.

Haraway (1991) usa la metáfora de la vista para criticar la característica de la ciencia tradicional de pretender que la mirada es omnisciente, que se tiene todo el conocimiento o la verdad sobre algo. Y, en cambio, aboga por la parcialidad, por la mirada situada, por el tartamudeo. No se trata de mirar desde ningún lugar en concreto para describir un conocimiento universal, sino de ocupar una posición para mirar desde ahí y obtener un conocimiento parcial, un conocimiento encarnado, un conocimiento que busca siempre conectar con otras/os. En palabras de la autora:

El feminismo ama otra ciencia: las ciencias y las políticas de la interpretación, de la traducción, del tartamudeo y de lo parcialmente comprendido. El feminismo trata de las ciencias del sujeto múltiple con (como mínimo) doble visión. El feminismo trata de una visión crítica consecuente con un posicionamiento en el espacio social generizado no homogéneo (Haraway, 1991: 336).

Así, desde nuestra lectura, Haraway (1991) sienta las bases para poner en práctica la epistemología feminista desde cualquier tipo de conocimiento que se busque. No hace falta que éste gire en torno a las mujeres ${ }^{1}$. Conocimientos situados también es una propuesta epistemológica muy adecuada para hablar de investigación activista -como iremos abordando a través de este texto.

\section{NUEVOS SUJETOS, NUEVAS APROXIMACIONES}

La emergencia de los movimientos altermundistas -a finales de los años noventa- y la tercera ola del feminismo coinciden en el tiempo (y no por casualidad) con la progresiva precarización de la investigación social, en especial en los países centrales del capitalismo (Virno, 2004; Federici, 2010).

Los nuevos movimientos globales comienzan a gestarse a finales de la década de la década de 1980 y principios de los años 1990, pero tienen sus grandes hitos con el alzamiento zapatista de 1994 y la contracumbre de Seattle en 1999, que supuso un punto de inflexión en relación con la visibilidad de estos movimientos.

${ }^{1}$ Hay otros trabajos que usan la epistemología feminista y que se basan concretamente en los conocimientos situados. Ver, por ejemplo: Biglia (2005); Araiza (2009); Piazzini (2014); Fulladosa-Leal (2015), entre otros. 
Aparecen, por lo tanto, después de la segunda oleada del movimiento feminista, y en coincidencia con la institucionalización -a través de las políticas públicasde parte de este feminismo de los años 1960. Precisamente, los movimientos por la justicia global coinciden en el tiempo con la emergencia de una tercera ola del feminismo que surge como crítica a algunos elementos del feminismo de la segunda ola, tales como la constatación de que no existe un único modelo de mujer, sino que existen múltiples sujetos femeninos determinados por cuestiones sociales, étnicas, religiosas, nacionales y de orientación sexual (Walker, 1995).

Las últimas décadas del siglo XX aportan también otra paradoja desde el punto de vista de la igualdad de género. Así, si bien se generalizan políticas públicas para garantizar la igualdad legal entre hombres y mujeres -e, incluso, políticas de discriminación positiva hacia las mujeres-, esto se da en un contexto de retroceso de los Estados del Bienestar y de dominio de la globalización neoliberal, frente a la cual las mujeres serán la principal víctima (Vivas, 2014). De esta forma, el incremento de la pobreza femenina en el mundo (Buvinic, 1998; Perez-Orozco, 2003) ${ }^{2}$ y de la violencia machista ${ }^{3}$, por ejemplo, conviven con un discurso políticamente correcto por parte de las instituciones y de algunas leyes que no atacan las raíces principales del patriarcado (González, 2014).

En consecuencia, en el interior de estos movimientos globales, veremos aflorar unas prácticas y discursos feministas, que vinculan la lucha contra el patriarcado con la lucha contra el capitalismo y el Estado, pues las consideran formas institucionales de legitimación de la dominación masculina.

Por otro lado, la eclosión de este nuevo ciclo de movilización mundial y de la tercera oleada del feminismo han acabado impactando inevitablemente en grupos de investigación social que desde dentro y fuera de la academia se plantean la necesidad de avanzar en el conocimiento aplicado hacia la trasformación social emancipadora (e. g., Col-lectiu Investigacció, 2005). Esta nueva generación de investigadores/as ha sufrido en sus cuerpos los rigores del neoliberalismo y de las políticas de ajuste estructural, en forma de trabajos precarios e inestables (Tamez y Pérez, 2009; Cuninghame, 2008; Sevilla, 2010). Son jóvenes que combinan su labor académica con trabajo eventual o a tiempo parcial en hostelería, tiempo libre u otros campos distintos a la universidad.

Además, estos investigadores/as -entre los que nos incluimos- no consideran al activismo solamente como un ámbito de estudio, sino que han sido o son activistas comprometidos en movimientos sociales, de manera que el activismo forma parte de su cotidianidad. Diversos encuentros entre investigadores y activistas desde finales de los noventa en la Universidad de Manchester

${ }^{2}$ El término "feminización de la pobreza" se introdujo en el vocabulario sobre el desarrollo a partir los años noventa, sobre todo con la constatación que se hizo en la IV Conferencia de Naciones Unidas sobre la presencia de un 70\% de mujeres entre los pobres del mundo (Tortosa, 2009).

${ }^{3}$ El término feminicidio (Lagarde, 2006) bien nos puede servir para conceptualizar esta sangrienta realidad. Por poner algunos datos recientes: según datos del Ministerio de Sanidad, Servicios Sociales e Igualdad del Gobierno de España, 800 mujeres fueron asesinadas por sus parejas o exparejas entre 2003 y 2015 en España, mientras que 26267 mujeres han sido asesinadas entre 2000 y 2014 en México (Aguilar, 2016). 
(Encuentro Internacional de Protesta Popular), en Barcelona (Jornades de Recerca Activista, en 2004), en Amsterdam (Encuentro Internacional auspiciado por el colectivo CASA, Cultural Analisys Summer Academy, también en 2004) y en redes temáticas y talleres de los Foros Sociales Europeos. Mundiales y de Educación, constataron la existencia de este nuevo sujeto. La nueva investigadora activista no es equiparable ni al experto académico en movimientos sociales, ni al intelectual comprometido, ya que su cuerpo ha sido atravesado por un contexto de precarización, que se ha traducido en inestabilidad laboral, curriculum no lineal y diverso, migración o exilio económico, entre otros. Este investigador activista prioriza los procesos colectivos y cooperativos de producción de conocimiento, debate y autoformación, frente al individualismo competitivo hegemónico en el mundo académico.

Consideramos que los nuevos sujetos emancipadores de finales del siglo XX y principios del XXI, como el feminismo de la tercera ola o los movimientos sociales por la justicia global, podrían beneficiarse con un abordaje investigador desde perspectivas activistas, por la propia naturaleza contestataria de los mismos, y porque los procesos contra los que luchan han afectado de pleno en los propios investigadores sociales activistas, que se encuentran por ese mismo motivo en una posición epistemológica privilegiada para comprender dichos procesos.

\section{LOS CONOCIMIENTOS SITUADOS Y LA INVESTIGACIÓN ACTIVISTA FEMINISTA}

Los conocimientos situados pueden ser compatibles con técnicas de investigación más críticas. En este apartado, analizaremos tres momentos y tradiciones, hasta cierto punto diferentes, pero que convergen en algunos puntos. Primero, hablaremos de la Investigación Acción Participativa (IAP), la cual parte de la necesidad de reconocer que la ciencia social debe comprometerse con la transformación social, económica, política y cultural de su entorno (Villasante, 1998; Jiménez-Domínguez, 1994). Es decir, la ciencia social se aproximaría a una praxis emancipadora. Esta tradición, si bien parte de la psicología social de Kurt Lewin, tiene un origen y una tradición arraigada en América Latina.

Para entender la IAP, Ander-Egg (1990) propone analizar los tres términos de que se compone la Investigación-Acción-Participativa. Así, en tanto "investigación", se trata de un procedimiento reflexivo, sistemático, controlado y crítico cuya finalidad es estudiar algún aspecto de la realidad. Como "acción" significa que la forma de realizar el estudio es ya un modo de intervención. Y, por último, como "participación" es una actividad en cuyo proceso están involucrados tanto los investigadores (equipo técnico o agentes externos) como la población destinataria del programa, quienes dejan de ser simples objetos de investigación, y se convierten sujetos activos que conocen y transforman su realidad. Hasta aquí, puede decirse que la IAP es suficientemente compatible con la propuesta de los conocimientos situados.

Por otra parte, la Investigación Activista o Investigación Militante también 
ofrece una perspectiva distinta a la investigación tradicional, la cual parte de la politización de quien investiga y de la participación activa en el colectivo que se estudia. Como la IAP, puede tener huellas marxistas. Sin embargo, es una aproximación más actual, en la que encontramos desarrollos de la escuela de Denzin y las metodologías cualitativas desarrolladas en la Universidad de Illinois a inicios del año 2000. Esta aproximación -dice Choudry (2014)- conlleva decidir, de acuerdo con el contexto, los métodos específicos. "No son solo postulados teóricos los que desarrolla la investigación activista en una relación dialéctica con respecto a su práctica, sino que también lo hace con las metodologías que usa" (Choudry, 2014: 80) ${ }^{4}$. Por su parte, Zavos \& Biglia afirman lo siguiente:

Se producen diferentes conocimientos en locaciones múltiples y hace falta reconocerlos, así como comprometerse con ellos, si queremos subvertir las relaciones desiguales que aún existen. Esto es exactamente lo que un análisis reflexivo y situado puede ofrecer en aras de politizar la investigación (Zavos \& Biglia, 2009: 155).

Es decir que aquí también hay una búsqueda de compromiso tanto teórico como político. En su estudio de caso, Alexandra Zavos comparte unas reflexiones sobre su investigación etnográfica:

Me enrolé en lo que llamaría investigación "activista", lo cual significa indicar mi involucramiento directo en la producción de mi campo/tema de investigación, así como mi posicionamiento como observadora externa y como miembro del grupo con el comparto prácticas. Quise estudiar, contribuir e influir al mismo tiempo que yo misma era influida por ellos (en Zavos y Biglia, 2009: $160)^{6}$.

En efecto, Investigación Activista conlleva pensar no sólo en el compromiso de quien investiga, sino en el conocimiento desde las relaciones de subordinación. Patricia Botero lo explica así:

Sustentar las militancias epistémicas o las militancias en la investigación conlleva a entablar la discusión propiamente dentro del campo del conocimiento de las ciencias y hacer una crítica a la subordinación que se ha establecido entre saber y conocimiento [...] (Botero, 2012: 33).

4 "It is not only theoretical frameworks that activist researcher develops in a dialectical relationship to their practice, but also the methodologies that they use" (Choudry, 2014: 80)

5 "Different knowledges are produced in multiple locations and these need to be acknowledged and engaged with if we are to challenge existing relations of inequality. This is precisely what a situated, reflexive analysis can offer towards a politization of research" (Zavos \& Biglia, 2009: 155).

6 'I engaged in what I would call 'activist' research, by which I mean to indicate my direct involvement in and production of my topic/field of research, my placement not as observing outsider but as member of the group, whose practices are as well as my own. I wanted to study, contribute to, and influence at the same time as I myself was influenced by them" (in Zavos \& Biglia, 2009: 160). 
Es decir que dicha apuesta, a diferencia de la IAP, tiene una posición más crítica con respecto a la producción de la ciencia misma, así como el uso de las metodologías para producirla. Por esta razón, nos parece que es aún más compatible con la idea de los conocimientos situados.

Pero aún nos gustaría ir un poco más allá. Por tanto, recuperamos y apostamos por la IAF, la cual guarda relación con la IAP y su compromiso por el cambio social, así como con la Investigación Activista y el claro posicionamiento de quien investiga una determinada realidad social. Así, dice Karina Fulladosa-Leal que la IAF:

[...] comparte base epistémica [...] a partir de considerar una posición situada, parcial y (con) sentido a la responsabilidad de lo que generamos y producimos. Así, nos encontramos en relación con otras, donde podemos crear a partir también de compartir nuestra historia y vivencia, politizando nuestra vida con las activistas en un espacio que nos reúne con un objetivo común [...] (Fulladosa-Leal, 2015: 122).

Podemos encontrar estos elementos en la IAP o en la Investigación Activista. Sin embargo, la IAF pretende ir más allá y asumir los postulados de los conocimientos situados, así como otras ideas propias de Haraway (2003), quien reconoce el "vínculo como unidad mínima de análisis". Por tanto, la afección (mutua) en la IAF es central. Barbara Biglia plantea once puntos que conforman la IAF: 1) el compromiso para el cambio social; 2) la ruptura de la dicotomía publico/privado; 3) la relación interdependiente entre teoría y práctica; 4) el reconocimiento de una perspectiva situada; 5) la asunción de responsabilidades; 6) la valoración y el respeto de las agencias de todas las subjetividades; 7) la puesta en juego de las dinámicas de poder que intervienen en el proceso; 8) una continua abertura a ser modificadas por el proceso en curso; 9) la reflexividad; 10) lógicas no propietarias del saber; y 11) la redefinición del proceso de validación del conocimiento utilizando diferentes métodos (Biglia, 2005).

Por supuesto que algunas de estas intenciones ya se observaban tanto en la IAP como en la Investigación Militante. Sin embargo, pensamos que algunas de las propuestas del endecálogo pasan necesariamente por la reflexión feminista, tales como: ruptura de la dicotomía publico/privado (preocupación frecuente en el feminismo); reconocimiento de una perspectiva situada (conocimientos situados); valoración y el respeto de las agencias de todas las subjetividades (reflexiones sobre la sujeción con Butler, 1997); puesta en juego de las dinámicas de poder que intervienen en el proceso (lecturas feminista de Foucault); continua abertura a ser modificadas por el proceso en curso (flexibilidad que enarbola Fulladosa-Leal, 2015); la reflexividad (difracción en Haraway, 1997); redefinición del proceso de validación del conocimiento utilizando diferentes métodos (testigo modesto mutado en Haraway, 1997). Es así que consideramos que hemos puesto en práctica algunos de estos puntos, a través de diferentes investigaciones, las cuales nos han ayudado a pensar la teoría y la práctica. Hablaremos de ellas a continuación. 


\section{CONOCIMIENTOS SITUADOS... EXPERIENCIAS CONCRETAS}

Este apartado resume algunas de las investigaciones en las que las autoras hemos participado -en distintos momentos de nuestra carrera académica- con el enfoque de la Investigación Activista. No tiene la intención de convertirse en un sumario de estas experiencias de investigación. Más bien, las analiza desde los planteamientos de la IAF y establece una suerte de diálogo entre pares académicos que -esperamos- nos ayude a visibilizar encuentros y a explicar algunos de los motivos por los cuales apostamos por ésta. En concreto, el apartado 4.1 se refiere a las experiencias de Robert González, el 4.2 a las de Alejandra Araiza y el 4.3 es el puente de análisis epistemológico y metodológico.

\subsection{INVESTIGACIÓN ACTIVISTA EN MOVIMIENTOS SOCIALES DE BARCELONA: EXPERIENCIAS CON PRO-VIVIENDA Y OKUPAS}

Desde el año 2003, cuando un grupo de investigadores activistas de Barcelona nos constituimos en el Col.lectiu Investigacció, he tenido la intención de vincular investigación y activismo, y de generar conocimiento colectivo y útil para los movimientos sociales (González, Martí, Alfama y Fuster, 2005). Por ejemplo, en 2005 participé como investigador-facilitador en un balance conjunto entre activistas, políticos de izquierdas y académicos del ciclo de luchas contra la globalización neoliberal y contra la guerra (Vilaregut y González, 2006). También en un estudio de la evolución del movimiento estudiantil catalán en el periodo 1993-2013, las autoras de la investigación habíamos participado como activistas del movimiento en diversos períodos (1993-2004 y 2005-2009, respectivamente) (González y Benítez, 2014). Estas primeras experiencias carecían todavía de una sistematización suficiente desde la óptica de la IAF y expresaban una voluntad de acercar los espacios activistas y académicos que no llegó a materializarse más que en momentos puntuales.

Sin embargo, en los últimos años he podido participar de investigaciones activistas que sí pueden ser sistematizadas desde la apuesta metodológica y epistemológica que defendemos en este artículo. Voy a analizar dos que parten de mi implicación con mi sujeto de estudio principal: el movimiento okupa. Se trata de un estudio sobre la Plataforma de Afectados por las Hipotecas (PAH, 2014-2017) y de la creación y evolución de un espacio de investigación activista, el Squatting Europe Kollective (SqEK, 2009-2017).

La investigación sobre las PAH es una etnografía de las Plataformas de Afectados por las Hipotecas de Barcelona, Sabadell y Terrasa, realizada con Oriol Barranco (UAB) y Marta Llobet (UB). Esta investigación era un subproducto del proyecto "El Movimiento Okupa en Europa, Prácticas y Contextos Sociales" (MOVEKEUR), coordinado por Miguel Martínez-López (UCM). La aproximación metodológica incluyó la participación en asambleas y en acciones de las Plataformas, tales como manifestaciones, acompañamientos o protestas 
ante entidades bancarias, durante más o menos 1 año, entre febrero de 2014 y febrero de 2015. Esta observación participante quedó recogida en las notas de campo, al tiempo que nos permitió seleccionar a las 14 personas con las que posteriormente realizaríamos entrevistas de trayectoria de vida en profundidad. (Barranco, González y Llobet, 2016).

Desde el primer día en que llegamos a las tres asambleas locales, la gente del movimiento nos acogió con protocolos ya pensados para recibir a investigadores, los cuales incluían nuestra participación e involucración en asambleas y acciones. Por otro lado, se tomó el compromiso -por parte de los investigadores- de realizar talleres de devolución de los resultados, que pudieran ser útiles para los activistas de estas tres plataformas, mismos que están previstos para el último trimestre de 2017. La tardanza en el proceso de devolución, debida a motivos vitales y biográficos de los propios investigadores (paternidad, migración, movilidad laboral) da cuenta también de la condición precaria del investigador-activista. El alargamiento del proceso no ha sido del todo negativo, ya que nos ha permitido compartir materiales y reflexiones con trabajos similares en Madrid.

El proyecto MOVEKEUR está estrechamente vinculado a un espacio de investigación militante que existe desde el año 2009 -en el ámbito de los investigadores y activistas okupas-: el Squatting Europe Kollective (SqEK). Esta red tiene como objetivo producir conocimiento fiable y específico sobre el movimiento okupa, pero no sólo como un fin en sí mismo, sino también como un recurso de acceso público para los mismos okupas y los activistas en general.

En su primer documento público, SqEK se situaba dentro de la investigación activista. En primer lugar, los enfoques transdisciplinares y comparativos son la base de este proyecto. El grupo es un colectivo transnacional cuyos miembros representan una gran variedad de campos y disciplinas necesarias para entender los temas asociados con la okupación. Para realizar rigurosos análisis críticos del movimiento okupa son relevantes sus contextos (históricos, culturales, espaciales, políticos y económicos) así como la involucración de los propios activistas en las investigaciones y la devolución de los resultados de la investigación al propio movimiento y a la sociedad en general. Con estas prácticas investigativas, se pone en juego la tradicional dicotomía entre los investigadores-activistas y sus sujetos/objetos de estudio, al tiempo que se favorece una aproximación colaborativa y dialógica en la producción del conocimiento (SqEK, 2010). También podríamos clasificar esta experiencia dentro del ámbito de la co-investigación (Peña-Cuanda y Esteban-Guitart, 2013).

Hasta la fecha, SqEK ha organizado dieciséis conferencias en diversas ciudades, como Madrid, Milán, Londres Berlín, Ámsterdam, Copenhague, Nueva York, Paris, Roma Barcelona, Rotterdam o Praga. En estos encuentros de SqEK, diversos activistas e investigadores okupas presentan los resultados de sus investigaciones, todo ello alternado con experiencias puramente activistas, debates entre militantes y académicos, y con la implicación de diversos centros sociales okupados de las ciudades anfitrionas. En algunos casos, se participó durante el encuentro de acciones reivindicativas como 
okupaciones, manifestaciones o concentraciones. En SqEK se han realizado diversas investigaciones y publicaciones tanto académicas como divulgativas, entre las que destaca un mapa interactivo de la okupación en Europa, donde gracias al trabajo de académicos y activistas se ha podido realizar un estudio socio-histórico-espacial de este movimiento social presente en Europa desde los años 60. Por otro lado la convivencia en este colectivo de activistas y académicos ha suscitado un fuerte diálogo entre un sector precario de la academia, investigadores más consolidados y activistas del movimiento okupa. Por ejemplo, en los últimos tres encuentros (Barcelona, 2015, Rotterdam 2016, Praga 2017) se han suscitado controversias sobre la "academización de SqEK" o sobre cuál debe ser la relación entre los asistentes a las conferencias y el activismo local (por ejemplo si se pueden realizar acciones directas del movimiento local con los participantes en la conferencia o si no existe un cierto peligro de banalización de estos congresos como una suerte de "turismo académico revolucionario"). Son cuestiones que han apelado directamente a la identidad y la razón de ser de SqEK. También se ha planteado -debido a las dinámicas transcontinentales- la necesidad de superar el marco europeo. Fruto de estos debates en el último encuentro que tuvo lugar en el Centro Social Autogestionado Klinica de Praga, se ha propuesto un cambio de nombre, que mantendría la siglas pero daría mejor cuenta de la actual composición y funcionamiento de este espacio: Squatting Everywhere networK.

La experiencia de SqEK, pero también la investigación sobre las PAH, coinciden en su compromiso para el cambio social, ya que en ambos casos los investigadores nos involucramos en movimientos que persiguen la mejora de las condiciones de vida de la ciudadanía o cambios profundos en el sistema económico y social hegemónico. En el caso de SqEK, la implicación de los académicos en el activismo okupa es una muestra de la ruptura de la dicotomía publico/privado. Los debates suscitados en SqEK sobre la academización o las condiciones de implicación de los asistentes a una conferencia en acciones directas de los movimientos locales anfitriones son muestras de interdependencia entre teoría y práctica, así como nuestra participación, en el marco de la etnografía, en acciones directas de las PAH. El reconocimiento de una perspectiva situada se refleja -aunque de forma indirecta- en el caso de SqEK, ya que las investigaciones parten de un sector del activismo okupa. Por lo que se refiere a las $\mathrm{PAH}$, los tres investigadores involucrados hemos participado en marchas y acciones en favor del acceso universal a una vivienda digna. La asunción de responsabilidades se da en ambos casos, en especial en la red SqEK donde se combina la investigación con la militancia en proyectos de centros sociales autogestionados. En las PAH se asumió la responsabilidad de participar junto con el sujeto de estudio en acciones de desobediencia civil durante el año que duró la etnografía.

La valoración y el respeto de las agencias de todas las subjetividades están muy presentes en la dinámica asamblearia de la red SqEK y podrán darse en la investigación de las PAH a finales de 2017 en los talleres de devolución. La puesta en juego de las dinámicas de poder que intervienen en el proceso 
se ha debatido ampliamente en SqEK, pues se ha abordado la cuestión de la academización y se ha problematizado la actitud extractivista de ciertos investigadores que desaparecen en cuanto han terminado sus tesis o artículos específicos. En las PAH, los investigadores adoptamos siempre una actitud de escucha y de implicación en las tareas cotidianas de las asambleas. Tanto la investigación sobre las PAH como el espacio SqEK, se han tenido una continua abertura a ser modificadas por el proceso en curso y cierta reflexividad, aunque no siempre es fácil combatir con las preconcepciones de sesgo positivista que incorpora muchas veces el quehacer científico. Los productos académicos y de divulgación de SqEK siguen lógicas no propietarias del saber. Finalmente, la redefinición del proceso de validación del conocimiento se está haciendo en el caso de las PAH al compartir las entrevistas cualitativas con investigadores que han realizado estudios similares en Madrid, mientras que en SqEK es una constante y se puede comprobar en muchas de sus publicaciones, que suelen incluir análisis comparativos realizados de forma conjunta por investigadores y activistas de países distintos, con formaciones académicas y tradiciones militantes diversas (Martínez, 2017).

\subsection{LA EXPERIENCIA ETNOGRÁFICA CON LAS FEMINISTES INDIGNADES DE BARCELONA}

La etnografía con las Feministes Indignades (FI), un colectivo surgido como comisión del 15M en Barcelona en 2011, fue parte de una investigación, que llevaba por nombre "Memory, Youth, Political Legacy and Civic Engagement" (MYPLACE). Se trataba de un proyecto amplio, cuyo foco de interés era la participación y el compromiso político de los/as jóvenes y el cual se realizó en 14 países europeos simultáneamente.

La etnografía de las Feministes Indignades se llevó a cabo entre dos personas, Mariona Ferrer (la coordinadora de la investigación en Cataluña, en la Universitat Pompeu Fabra) y Alejandra Araiza. Cuando llegamos al equipo, el contacto con las FI ya se había hecho a través de una alumna de la UPF, lo que facilitó el comienzo del trabajo de campo. Por acuerdo de asamblea, las FI tenían una militante (con formación académica próxima a la teoría feminista) como encargada de revisar cualquier proyecto o intento de investigar este colectivo. Esta compañera encargada dio su visto bueno. Y, después de haberse discutido en la asamblea, se aceptó que hiciéramos la etnografía.

Ésta siguió las directrices de una observación participante-respetuosa. Realizamos un diario de campo conjunto conforme íbamos participando en asambleas y diversas acciones que emprendían las FI, así como 19 entrevistas a profundidad a distintos perfiles de militancia, la mayoría muy activas en el colectivo, una militante casual y una simpatizante. El guion de la entrevista abordaba temas relacionados con la experiencia militante, la historia y funcionamiento del colectivo, la memoria, la vida cotidiana, entre otros. Aunque estaba calculado que podría hacerse de manera intensiva en tres meses, ello no 
fue posible si se pretendía hacer un proceso cuidadoso. Por tanto, la etnografía duró 8 meses, de octubre de 2012 a junio de 2013.

Así, en el fluir de la etnografía, aunque nos sentíamos muy acogidas, por momentos también nos sentíamos interpeladas. Desde el principio, llamó mi atención leer en el acta del 4 de octubre de 2012 -cuando se decidió si aceptaban nuestra presencia o no- que algunas de las compañeras opinaron que la investigación no estaba pensada desde una perspectiva feminista. Ahí estuvo el primer dilema: siendo la autora de epistemología feminista, tenía que realizar una investigación que no había diseñado, aunque, por otro lado, cabe mencionar que sí era respetuosa y acorde a las teorías que trabajamos. Así, aunque se hubiera concebido la investigación desde los conocimientos situados de Donna Haraway (1991) o hubiese pensado la aproximación desde una etnografía más performativa (Denzin, 2003) que rozase los límites entre la etnografía misma y la IAF, tuve que trabajar con lo que teníamos a la mano (Araiza, 2015).

En esta sección, nos gustaría presentar algunas reflexiones que nos ayuden a pensar en la utilidad del endecálogo de Barbara Biglia (2005). Desde nuestra lectura, no se trata de seguir estos once puntos como prescripciones para emprender una IAF. En el caso de la etnografía con las FI, muchos de estos puntos fueron llevados a cabo por el mismo vínculo que se estableció con nuestros sujetos de investigación y el diálogo con este movimiento social concreto.

De esta forma, en el diseño original de la investigación -pensada desde los estudios culturales británicos- había simpatía por los movimientos sociales que se estudiaron en los 14 países. Se buscó que quienes hicieran las etnografías fueran militantes o simpatizantes de los mismos. Por lo que el compromiso para el cambio social que promueven estos movimientos sí estaba presente. Si bien no se abrazaba una perspectiva situada, sí que era más o menos visible la posición política de quienes hacíamos las etnografías.

A lo largo del proceso, como ejercicio reflexivo y en diálogo constante entre las dos personas que hacíamos la etnografía de las FI, nos íbamos percatando de nuestra propia posición como mujeres, madres de niñas pequeñas. Cuando las Feministes Indignades debatían sobre la sostenibilidad de la vida y del cuidado, reflexionábamos sobre la experiencia personal, del ámbito privado, al tiempo que participábamos en las acciones que buscaban la irrupción del espacio público. A menudo estas fronteras se diluían en el trato con ellas.

Otro de los puntos clave en el diálogo con los movimientos sociales es la relación entre la teoría y la práctica. Cabe mencionar que la mayoría de las militantes de FI eran mujeres con preparación académica, pero que tenían trabajos precarios, muchos de ellos relacionados con la perspectiva de género. Nosotras mismas estabamos en esta posición. Asimismo, en los debates de las propias asambleas, aunque se hablase de acciones concretas, también había un nivel elevado, en el que la teoría se diluía en la práctica.

Por otra parte, durante todo el proceso nos fuimos sintiendo interpeladas por el propio colectivo, lo que nos lleva a pensar los puntos sobre la asunción de responsabilidades; la valoración y el respeto de las agencias de todas las subjetividades; así como la puesta en juego de las dinámicas de poder. En primer 
lugar, estuvo el aspecto de la participación. Estábamos como observadoras en las asambleas, pero no nos atrevíamos a participar. Y un día, pasó lo siguiente:

Media hora más tarde -como suele suceder-, empezó la reunión con 10 asistentes (más tarde acabarían siendo 15). [...] Estaba una de las dinamizadoras (la otra llegaría más tarde), alguien más se propuso para tomar palabras, pero ¿quién tomaría notas para hacer el acta? Que lo hagan las investigadoras -dijo una-. Yo me reí y la chica vasca dijo: 'que no es broma'. Lo pactamos un poco con Mariona y les dijimos que sí, que tomaríamos notas. [...] De alguna forma, ellas nos están pidiendo que, si estamos ahí, ocupando un lugar en la sala, presenciando las reuniones, participemos (Notas del diario de campo, 28 de noviembre de 2012).

A raíz de este episodio, entre la coordinadora y yo fuimos pensando en la posibilidad de hacer una devolución. Así, les ofrecimos a las FI un documento de devolución justo en un periodo en que ellas estaban pensando en balances internos (alrededor de los meses de marzo y abril de 2013). Y les pareció buena idea. A partir de entonces, aunque no participamos mucho en los debates de la asamblea, ocupamos una posición y asumimos una responsabilidad.

La etnografía se fue desarrollando y -a lo largo de la misma- se fue construyendo un vínculo en el que se intentaba respetar a todas las agencias o subjetividades. Particularmente, las entrevistas fueron una pieza clave en la construcción de este vínculo. Así, a medida que íbamos teniendo estos momentos más privados y de mayor complicidad con ellas, nuestra propia presencia en la asamblea era diferente. Cada vez, nos sentíamos menos "intrusas". Y, hacia el final, nos atrevimos a tomar la palabra en alguna asamblea.

En cuanto a las dinámicas del poder, creo que nuestro reparo para participar también venía de no querer ocupar la posición del saber-poder, de quienes vienen de un espacio de conocimiento a querer explicar el movimiento. Pero, al mismo tiempo, íbamos recopilando una información que podía ser útil para el propio movimiento. Por eso el documento de devolución fue tan importante para este proceso, pues pusimos esto a su disposición. El texto -basado en los diarios de campo y las entrevistas- explicaba en qué consistía la etnografía, los periodos que abarcaba y para qué queríamos la información. Asimismo, explicábamos lo que nosotras habíamos observado. Y organizamos las voces de sus propias participantes (extraídas de las entrevistas sin explicitar nombres) de la siguiente forma: 1) los logros del colectivo, 2) aspectos a destacar, lo que más gustaba de FI, 3) las acciones exitosas y que más se recordaban, 4) opiniones sobre las asambleas y sus dinámicas, 5) recomendaciones para seguir trabajando y enganchando con ejes y objetivos y 6) cómo compaginaban el feminismo y la vida cotidiana. Finalmente, en un apartado de conclusiones, les sugeríamos trabajar tres bloques: a) vidas vivibles (la búsqueda de la sostenibilidad de la vida), b) dar especial énfasis a la reflexión decolonial y la inmigración, así como al tema de la desigualdad social y c) sexualidad, transexualidad, derecho al propio cuerpo y otras maternidades. Esta reagrupación de bloques era nuestra sugerencia de lo que veíamos que eran los intereses reales de las entrevistadas y las prioridades 
de debate en las asambleas.

Dicho documento pasó primero la revisión por la misma persona que había evaluado el proyecto de investigación. A ella le pareció correcto. Así, entregamos varias copias en una sesión muy emotiva que cerraba el vínculo que habíamos tejido entre las dos etnógrafas y el colectivo de las Feministes Indignades.

El ejercicio de la devolución, que no estaba diseñado desde el principio, también es una muestra de cierta abertura a ser modificadas por el proceso en curso, pues lo hicimos a pesar de que no era parte del guion y lo hicimos porque nos sentimos interpeladas. Por nuestra parte, intentamos llevar a cabo el ejercicio de la reflexividad a través de las notas del diario de campo y en los diálogos que entablamos con la coordinadora de la investigación, quien también realizaba la etnografía. Y creo que en los resultados hay mucho de lo que nos fue pasando a nosotras durante esta etapa, producto del vínculo con las FI. Por eso creo que la IAF puede ser una herramienta metodológica muy potente para construir conocimiento en diálogo con un movimiento social.

\subsection{CONVERSACIONES COMPARTIDAS EN LA EPISTEMOLOGÍA}

Pensamos que la metodología (la teoría del método) no debe considerarse como una serie de reglas a seguir, sino que debe conformar distintas prácticas que emanan del quehacer científico y del diálogo entre pares. Ése es el ejercicio que hemos intentado llevar a cabo en este artículo. La hoja de ruta han sido los conocimientos situados de Haraway (1991) y la propuesta de IAF de Biglia (2005).

Las reflexiones que aquí presentamos forman parte de nuestra experiencia como investigadoras precarias de un contexto sociopolítico específico, así como de nuestra experiencia militante y/o simpatizante de algunos movimientos sociales (incluido el feminismo). La primera reflexión que nos convocó a escribir este texto es que compartimos el hecho de habernos sentido interpeladas -en el fluir de nuestras investigaciones- por los propios movimientos sociales y -de alguna manera- obligadas a entablar un diálogo con ellos. Esto concuerda con lo que Haraway (1991) define como conocimientos situados que, además de ser solidaridad en lo político, son conversaciones compartidas en la epistemología.

Como resultado de estas conversaciones, pensamos que para estudiar un movimiento social es conveniente seguir los planteamientos de la epistemología y la metodología feministas. No hace falta que el movimiento social en sí se enmarque en los límites del feminismo. Pensamos que el ejercicio de posicionamiento, de vínculo, de respeto por todas las agencias involucradas, así como del reconocimiento de las dinámicas de poder -que proponen estos planteamientos teóricos feministas- son deseables para la aproximación a diversos movimientos sociales.

El siguiente cuadro, sintetiza las principales características que encontramos de la epistemología feminista de los conocimientos situados, así como los puntos correspondientes a cada una de ellas del endecálogo de la IAF (Biglia, 2005). 
Resumimos estos planteamientos y los aterrizamos en ejemplos concretos de las experiencias investigadoras aquí presentadas. En la primer columna, hemos agrupado las características de la epistemología feminista de los conocimientos situados (Haraway, 1991) en seis bloques: I. Conocimiento parcial, el cual no pretende hacer generalizaciones; II. Posicionamiento político, el cual debe ser claro y explícito; III. Objetividad situada, que no busca la verdad universal, pero sí una verdad concreta y comprometida; IV. Conexiones parciales, vínculos que se construyen en el proceso, tanto con los sujetos de investigación como con otras agencias relacionadas; V. Difracción, la cual proyecta la mirada del otro pero desde el reconocimiento de la diferencia; y, por último, VI. Testigo modesto mutado, el cual proviene de la necesidad de objetividad que se construye al interior de una comunidad científica, pero que no apuesta por la universalidad androcéntricaoccidental-heteronormada, sino por la mera validación de conocimientos en una comunidad más plural (Haraway, 1997). En la segunda columna hemos asignado distintos puntos del endecálogo de Biglia (2005) a cada uno de los bloques. Ésta es sólo una lectura posible de esta relación entre epistemología y metodología. Y nos sirve para presentar, en la tercera columna, una síntesis no exhaustiva de la presencia de estas características en las experiencias de investigación analizadas.

\section{Cuadro 1. Síntesis de experiencias investigadoras}

\begin{tabular}{|l|l|l|}
\hline $\begin{array}{l}\text { EPISTEMOLOGÍA } \\
\text { FEMINISTA } \\
\text { CONOCIMIENTOS } \\
\text { SITUADS }\end{array}$ & $\begin{array}{l}\text { METODOLOGÍA } \\
\text { IAF }\end{array}$ & $\begin{array}{l}\text { EXPERIENCIAS } \\
\text { INVESTIGADORAS }\end{array}$ \\
\hline $\begin{array}{l}\text { I. Conocimiento } \\
\text { parcial }\end{array}$ & $\begin{array}{l}\text { 2. Ruptura público/privado } \\
\text { 7. Rel. Teoría-práctica } \\
\text { dinámicas de poder } \\
\text { 8. Abertura a ser } \\
\text { modificadas por el proceso }\end{array}$ & $\begin{array}{l}\text { PAH y SqEK: problemas de } \\
\text { vivienda } \\
\text { FI: sostenibilidad de la vida } \\
\text { SqEK: debate sobre la } \\
\text { academización } \\
\text { FI: incorporar prácticas al diseño } \\
\text { de investigación }\end{array}$ \\
\hline $\begin{array}{l}\text { II. Posicionamiento } \\
\text { político }\end{array}$ & $\begin{array}{l}\text { 1. Compromiso para el } \\
\text { cambio social } \\
\text { 5. Asunción de } \\
\text { responsabilidades }\end{array}$ & $\begin{array}{l}\text { PAH y SqEK: movimiento pro- } \\
\text { vivienda y movimiento okupa } \\
\text { (militancia/simpatía) } \\
\text { FI: feminismo y 15M (militancia/ } \\
\text { simpatía) } \\
\text { PAH y FI: ejercicios de } \\
\text { devolución }\end{array}$ \\
\hline $\begin{array}{l}\text { III. Objetividad } \\
\text { situada }\end{array}$ & $\begin{array}{l}\text { PAH y FI: se pensaron desde la } \\
\text { proximidad con el movimiento } \\
\text { SqEK: fronteras borrosas entre } \\
\text { investigadores y militantes }\end{array}$ \\
\hline
\end{tabular}




\begin{tabular}{|l|l|l|}
\hline $\begin{array}{l}\text { EPISTEMOLOGÍA } \\
\text { FEMINISTA } \\
\text { CONOCIMIENTOS } \\
\text { SITUADOS }\end{array}$ & $\begin{array}{l}\text { METODOLOGÍA } \\
\text { IAF }\end{array}$ & $\begin{array}{l}\text { EXPERIENCIAS } \\
\text { INVESTIGADORAS }\end{array}$ \\
\hline $\begin{array}{l}\text { IV. Conexiones } \\
\text { parciales }\end{array}$ & $\begin{array}{l}\text { 6. Valoración y respeto de } \\
\text { agencias }\end{array}$ & $\begin{array}{l}\text { SqEK: organización asamblearia } \\
\text { de activistas investigadores }\end{array}$ \\
\hline $\begin{array}{l}\text { V. Difracción } \\
\text { VI. Testigo modesto } \\
\text { mutado }\end{array}$ & $\begin{array}{l}\text { 10. Lógicas no propiedad } \\
\text { del saber } \\
\text { 11. Validación-triangulación }\end{array}$ & $\begin{array}{l}\text { Fq: diario de campo, (auto) } \\
\text { etnografía }\end{array}$ \\
\hline interactivas
\end{tabular}

Fuente: elaboración propia

Así, en cuanto a los puntos relacionados con el conocimiento parcial, estas investigaciones no fueron pensadas desde la epistemología feminista, pero sí se observaban algunos de sus principios. En este bloque, recogemos varios puntos del endecálogo de la IAF. En relación con la ruptura entre lo público y lo privado, sobresalen las tres experiencias de investigación, pues cuestionaban nuestras propias prácticas: las PAH y el movimiento okupa por nuestros propios problemas de acceso a la vivienda y FI por el cuestionamiento a la sostenibilidad de la vida. En estos espacios de movilización, es común encontrar reflexiones y prácticas que buscan subvertir la distancia existente entre la actuación en el espacio público (discursos y manifestaciones) con las tareas domésticas y del cuidado. Se intenta no darle más importancia a las primeras e invisibilizar las otras. Se procura no repartirlas en función del género. Y, aunque no siempre se logra, vemos que muchos de sus militantes realizan ambos tipos de tareas. Como investigadoras y como militantes hemos hecho lo propio.

Por lo que respecta a la relación interdependiente entre teoría y práctica, podemos decir que muchas reflexiones vienen de nuestro hacer, de nuestras prácticas encarnadas, las cuales nos llevaron a pensar en la teoría y a modificar nuestro ejercicio científico. En cuanto al reconocimiento de las dinámicas de poder que intervienen en el proceso, se podía observar en los distintos movimientos sociales que estudiamos y que intentaban subvertir las relaciones tradicionales, atravesadas por el poder (en especial, lo vimos en el movimiento okupa y FI como parte de los feminismos del 15M). Ello nos obligaba, en todo momento, a cuestionarnos nuestra propia posición de saberpoder como investigadoras. La apertura a ser modificadas por el proceso en curso la observamos, particularmente, en la etnografía con las FI, en la cual se fueron tomando decisiones sobre la marcha; se fue decidiendo nuestro nivel de implicación y, algunas veces, se asumieron tareas. 
El segundo bloque epistemológico, el posicionamiento político, abarca dos puntos del endecálogo de la IAF. En general, observamos en nuestras investigaciones un claro compromiso para el cambio social, pues los estudios de movimientos sociales, tales como: el movimiento pro-vivienda, el movimiento okupa, el feminismo o el 15M, los cuales en sí mismos proponen importantes cambios sociales y las investigadoras compartíamos ese compromiso. En cuanto a la asunción de responsabilidades, se puede decir que en todos los casos participamos y asumimos una posición militante con las responsabilidades que ello tenía y fuimos cuidadosas con la presentación de los resultados de cada investigación.

$\mathrm{Al}$ tercer bloque, objetividad situada, le corresponde el punto del reconocimiento de una perspectiva situada. Aunque no fueron investigaciones diseñadas desde estos planteamientos epistemológicos, sí que se partió de la proximidad con los movimientos y de una posición crítica a los planteamientos de la ciencia social tradicional -enmarcada en el positivismo.

El cuarto bloque, conexiones parciales, podemos poner el ejemplo de SqEK, en tanto que su dinámica asamblearia activistas/investigadores somete a continua revisión el trabajo académico y dota a los activistas de agencia para analizar y estudiar su propio movimiento social. El siguiente bloque, difracción, es bastante afín, pues tiene que ver con el vínculo que se construye con el sujeto de investigación, el cual estuvo particularmente presente en la etnografía con las FI, toda vez que en el diario de campo se intentaba recoger no sólo lo observado, sino también lo que nos iba pasando a nosotras en el proceso, cómo nos sentíamos interpeladas por el mismo colectivo.

El último bloque, testigo modesto mutado, recoge la apuesta de Haraway (1997) de no renunciar a la creación del conocimiento en espacios de comunidad, pero abogar por otras formas de validación colectiva del saber. Cabe señalar que, al no tener un diseño dentro de este marco, estos dos puntos no son claramente observables en nuestras experiencias de investigación. Sin embargo, el caso de SqEK sí que presenta algunos de estos elementos por la lógica no propietaria de sus publicaciones y la continua validación de las mismas por parte de los activistas que participan en esta red. Asimismo, aunque este artículo no es exactamente un ejercicio de triangulación, en el que intercambiamos datos para tener otras perspectivas de análisis, sí que compartimos reflexiones -surgidas de nuestras prácticas investigadoras-, las cuales nos sirven para validar algunos planteamientos epistemológicos y metodológicos.

\section{CONCLUSIONES}

No siempre la teoría y la práctica coinciden. Por lo general, los planteamientos teóricos sobre la investigación no suelen acompañarse de prácticas consecuentes. Sin embargo, pensamos que la investigación es una práctica viva que cambia constantemente y a la cual nos vamos adaptando. En el caso de las investigaciones sobre movimientos sociales aquí presentadas, hemos recogido -de forma explícita 
o implícita- gran parte de los principios del endecálogo de Barbara Biglia (2005). Por ello, consideramos que la IAF sería la opción metodológica más apropiada para trabajar con movimientos sociales.

En efecto, ubicamos nuestras investigaciones en la línea de los conocimientos situados y la epistemología feminista. Pensamos que el conocimiento debe ser reconocido en su multiplicidad y parcialidad. Creemos que el conocimiento debe producir conversaciones desde la difracción, es decir que no se trata de buscar el reflejo de la realidad social de manera transparente, sino de producir proyecciones desde la diferencia. Por tanto, apostamos por una IAF, la cual puede llevarse a cabo con distintos tipos de subjetividades, no sólo colectivos feministas o de mujeres. Creemos que es lo que hemos ido haciendo en estas investigaciones, aunque no haya sido inicialmente buscado. Tal parece que, en algunos momentos la práctica nos llevó a la teoría y no al revés. Y es que, en efecto, la relación entre ambas no debería ser unidireccional y jerárquica, sino interdependiente.

Cabe señalar que algunas limitaciones de este tipo de investigaciones son que no pueden ser del todo previstas ni replicables. Requieren una implicación previa de las investigadoras con el sujeto de estudio o una construcción cuidadosa del vínculo. Sin embargo, pensamos que esto es también su potencia, toda vez que renuncian a la pretendida universalidad científica y apuestan -en cambiopor un circuito universal de conexiones parciales que sea capaz de reconocer las diferencias (Haraway, 1991). Ese es el ejercicio que hemos intentado hacer.

Para finalizar, nuestra relación con los movimientos sociales como investigadoras activistas nos ha llevado a dos conclusiones que juntas son paradójicas, pero que reflejan bien la pluralidad del sujeto de los movimientos sociales. Así, en nuestros trabajos de campo nos ha sorprendido cómo algunos movimientos sociales actuales han incluido de forma auto-reflexiva y pragmática a la investigación social en su interior. Hallamos esto tanto en la investigación las Plataformas de Afectados por las Hipotecas de Barcelona, Terrassa y Sabadell -en la que participó Robert González-, como en la de las Feministes Indignades -en la que participó Alejandra Araiza-. Estos colectivos tenían todo un protocolo para afrontar la llegada de investigadores sociales. Este protocolo comprendía una bienvenida por parte de una comisión de investigación del propio colectivo y la obligatoriedad de implicarse en las actividades del movimiento, al menos por el tiempo que durara el trabajo de campo. De algún modo, nuestro enfoque partidario de la investigación activista nos facilitó mucho estas tareas. Sin embargo, en algunos movimientos sociales, como el de okupaciones, existen sectores alertan del peligro de academización de los espacios de investigación activista. De nuevo, teoría y práctica, con implicaciones a menudo complejas, pero siempre apasionantes. 


\section{REFERENCIAS}

Aguilar, R. (2016): "Feminicidio en México". En Animal Político, 5 enero 2015. Disponible en http://www.animalpolitico.com/blogueros-lo-que-quisodecir/2016/01/05/feminicidio-en-mexico/ [Consulta: 15/11/2016]

Ander-Egg, E. (1990): Repensando la Investigación-Acción-Participativa, Buenos Aires, Lumen.

Araiza, A. (2009): Conocer y ser a través de la práctica del yoga: una propuesta feminista de investigación preformativa (Tesis inédita de doctorado), Universidad Autónoma, Barcelona, España.

(2013): "De la política de la localización a los conocimientos situados. Notas para la creación de una ciencia feminista", en Liévano, M y Duque, M. (Comps.), Subjetivación femenina: investigación, estrategias y dispositivos críticos, pp. 163191, Monterrey, Ediciones UANL. Colección Tendencias.

(2015). En busca de la congruencia epistemológica. Una etnografía de las Feminsites Indignades en Barcelona. Boletín Edähi, 4(7). Recuperado de https://www.uaeh. edu.mx/scige/boletin/icshu/n7/a1.html

Barranco. O., González, R. y Llobet, M. (2016). "Del movimiento okupa a las PAH: cambios en las visiones e interpretaciones de la ocupación de viviendas" XII Congreso Español de Sociología, 30 de junio, 1-2 de julio de 2016, Gijón, España.

Biglia, B. (2005): Narrativas de mujeres sobre las relaciones de género en los movimientos sociales. (Tesis de doctorado, Universidad de Barcelona). Recuperado de https://www.academia.edu/313091/Narrativas de mujeres sobre las relaciones de $\mathrm{g} \% \mathrm{C} 3 \% \mathrm{~A} 9$ nero en los movimientos sociales.

Botero, P. (2012): "Investigación y acción colectiva -IAC- Una experiencia de investigación militante", Utopía y praxis latinoamericana, 57, pp. 31-47.

Butler, J (1997): Mecanismos psíquicos del poder. Teorías sobre la sujeción, Madrid, Cátedra/Universitat de València, 2001.

Buvinic, M. (1998): Mujeres en la pobreza: un problema global. Disponible en http:// www.iadb.org/sds/doc/769spa.pdf [Consulta: 13/11/2016]

Choudry, A. (2014): "(Almost) everything you wanted to know about activist research but were afraid to ask: what activist researchers say about theory and methodology", Contention: The Multidisciplinary Journal of Social Protest, 1(2), pp. 75-88.

Col-lectiu Investigacció. (2005): Recerca Activista i Moviments Socials. Barcelona, Viejo Topo/ Fundació Jaume Bofill.

Cuninghame, P. (2008): "EduFactory: precarización de la producción del conocimiento y alternativas", Bajo el Volcán, 7 (13), pp. 11-24.

Denzin, N. (2003): “The call to performance", Symbolic Interaction, 26 (1), pp. 187-207.

Federici, S. (2010). El trabajo precario desde un punto de vista feminista. Sin permiso. Disponible en http://www.sinpermiso.info/textos/el-trabajo-precario-desde-unpunto-de-vistafeminista [Consulta: 14/10/2016]

Fulladosa-Leal, K. (2015): "Creando puentes entre la formación y la creatividad: una experiencia de investigación activista feminista”, Universitas Humanística, 79, pp. 115-140.

González, R; Martí, M; Alfama, E. y Fuster, M. (2005): "Evolució dels moviments socials metropolitans: experiències comparades de treball glocal", en Col-lectiu Investigacció, Recerca Activista i Moviments Socials, pp. 155-168, Barcelona, Viejo Topo. 
González, R. (2014): "Feminismo y movimientos globales. Las activistas catalanas en el nuevo ciclo de luchas", en Mir, J. y Renom, M. (Eds.), Revoluciones en femenino. Escenarios entre el siglo XVIII y la actualidad, pp. 233-246, Barcelona, Icaria.

González, R. y Benítez, I. (2014): "El movimiento estudiantil catalán en el nuevo ciclo de luchas", Ánfora, 21, (37), pp. 101-128.

Haraway, D. (1991): “Conocimientos situados: la cuestión científica en el feminismo y la perspectiva parcial”, en Ciencia, cyborgs y mujeres. La reinvención de la naturaleza, pp. 313-346, Madrid, Cátedra/Universitat de València, 1995.

(1997): Testigo_Modesto@Segundo_Milenio. HombreHembra@_Conoce_ Oncoratón ${ }^{\circledR}$. Barcelona, Ediciones UOC, 2004.

(2003): The companion species manifiesto. Dogs, people and significant otherness, Chicago, Prickly Paradigms Press.

Jiménez-Dominguez, B. (1994): “Investigación ante acción participante: una dimensión desconocida", en Montero, M. (Coord.), Psicología social comunitaria, pp.112-138, Guadalajara, Universidad de Guadalajara.

Lagarde, M. (2006): "Del femicidio al feminicidio", Desde el Jardín de Freud. Revista de Psicoanálisis, 6, pp. 216-225.

Martínez, L. M., Biglia, B., Luxán, M., Fernández, C., Azpiazu, J. y Bonet, J. (2014): "Experiencias de investigación feminista: propuestas y reflexiones metodológicas", Athenea Digital, 14(4), pp. 3-16.

Marínez, M.A.,(ed) (2017): Urban politics and squatters'movments in Europe. Contexts, cycles and comparisons. London: Palgrave Macmilan.

Pérez-Orozco, A. (2005): "Feminización de la pobreza: Mujeres y recursos económicos", Materiales de reflexión, 3, pp. 2-15.

Peña-Cuanda, M. y Esteban-Guitart, M. (2013): "El estudio de las identidades desde un enfoque cualitativo. La multi-metodología autobiográfica extendida y los talleres lúdico-reflexivos", Empiria, Revista de Metodología de Ciencia Sociales, 26, pp. 175-200.

Piazzini, C. E. (2014): "Conocimientos situados y pensamientos fronterizos: una relectura desde la universidad", Geopolítica(s), 5(3), pp. 11-33

Sevilla, C. (2010): La fábrica del conocimiento. La universidad-empresa en la producción flexible, Barcelona, El Viejo Topo.

SqEK (2010): Squatting Europe Research Agenda, 1.0, disponible en https://sqek.squat. net/eng-squatting-europe-research-agenda-v-1-0/ [Consulta: 12/05/2016]

Tamez, S. y Pérez, J. F. (2009), "El trabajador universitario: entre el malestar y la lucha", Educação y Sociedade, 30 (107), pp. 373-387.

Tortosa, J. M. (2009), "Feminización de la pobreza y perspectiva de género", Revista Internacional de Organizaciones, 3, pp. 71-89.

Vilaregut, R. y González, R. (2006): "El ciclo de movilizaciones (2000-2005) y su valoración", en Grau, E. y Ibarra, P. (Coords.), La red en la encrucijada. Anuario de movimientos sociales 2005, pp. 33-41, Barcelona, Betiko Fundazioa- Icaria editorial.

Villasante, T. R. (1998): Cuatro redes para mejor-vivir, Buenos Aires, Lumen-Humanitas.

Virno, P. (2004): A grammar of the multitude: For an analysis of contemporary forms of life, New York, Semiotext(e).

Vivas, E. (2014): "Sin las mujeres no hay soberanía alimentaria", en Mir, J. y Renom, M. (Eds.), Revoluciones en femenino. Escenarios entre el siglo XVIII y la actualidad, pp. 219-232, Barcelona, Icaria.

Walker, R. (1995): To Be Real: Telling the Truth and Changing the Face of Feminism, New York, Anchor Books. 
Zavos, A. y Biglia, B. (2009): "Embodying Feminist Research: Learning from Action Research, Political Practices, Diffractions, and Collective Knowledge", Qualitative Research in Psychology, 6, pp. 153-172. 